CUBO A Mathematical Journal

Vol.20, $N^{\underline{Q} 02,(53-66) .}$ June 2018

\title{
Optimal control of a SIR epidemic model with general incidence function and a time delays
}

\author{
Moussa Barro, Aboudramane Guiro and Dramane Ouedraogo \\ Département de Mathématiques, UFR des Sciences et Techniques, \\ Université Nazi Boni, \\ Laboratoire d'Analyse Mathématique et d'Informatique (LAMI) \\ B.P. 1091 Bobo-Dioulasso, Burkina Faso. \\ mousbarro@yahoo.fr, abouguiro@yahoo.fr, dramaneouedraogo268@yahoo.ca.
}

\begin{abstract}
In this paper, we introduce an optimal control for a SIR model governed by an ODE system with time delay. We extend the stability studies of model (2.2) in section 2, by incorporating suitable controls. We consider two control strategies in the optimal control model, namely: the vaccination and treatment strategies. The model has a time delays that represent the incubation period. We derive the first-order necessary conditions for the optimal control and perform numerical simulations to show the effectiveness as well as the applicability of the model for different values of the time delays. These numerical simulations show that the model is sensitive to the delays representing the incubation period.
\end{abstract}

\section{RESUMEN}

En este artículo, introducimos un control óptimo para un modelo SIR gobernado por un sistema de EDOs con retardo temporal. Extendemos los estudios de estabilidad del modelo (2) en la sección 2, incorporando controles apropiados. Consideramos dos estrategias de control en el modelo de control óptimo, llámense: las estrategias de vacunación y tratamiento. El modelo tiene un retardo en el tiempo que representa el período de incubación. Derivamos las condiciones necesarias de primer orden para el control óptimo y realizamos simulaciones numéricas para mostrar la efectividad y también la aplicabilidad del modelo para diferentes valores de los retardos temporales. 
Estas simulaciones numricas muestran que el modelo es sensible a los retardos que representan el período de incubación.

Keywords and Phrases: SIR, general incidence, delays, optimal control, epidemic models, Hamiltonian.

2010 AMS Mathematics Subject Classification: 34K19, 34K20, 49K25, 49K30, 65N06, 90C90. 


\section{Introduction}

Mathematical modeling of population are often used to describe the dynamics of epidemic diseases. This is a fast growing research area and has been paying important roles in discovering relations between species and their interactions. There have been many variations such as classical epidemiological models [11. These models are based on the standard Susceptible-Infectious-Susceptible (SIS), Susceptible-Infectious-Recovered (SIR) and Susceptible-Exposed-Infectious-Recovered (SEIR) models, which are determined according to the difference on the method of transmission, nature of the disease, those with short/long incubation period, killer/curable diseases, etc, and the response of the individuals to it, for instance, gaining transient/permanent immunity, dying from the disease, etc. [6, 16. The main purpose of formulating a such epidemiological model is to understand the long-term behavior of the epidemic disease and to determine the possible strategies to control it. Differential equations, whether there are ordinary, delay, partial or stochastic are one of the main mathematical tools being used to formulate many epidemiological models. The focus in such epidemiological models has been on the general incidence at which people move from the class of susceptible individuals to the class of infective individuals.these general incidence have been modeled mostly by using bilinear and Holling type of functional responses [10, 12.

On the other hand, optimal control has extensively been used a strategy to control the epidemic outbreaks [ 8 . The main idea behind using the optimal control in epidemics is to search for, among the available strategies, the most effective strategy that reduces the infection rate to a minimum level while optimizing the cost of deploying a therapy or preventive vaccine that is used for controlling the disease progression [18. In terms of epidemic diseases, such strategies can include therapies, vaccines, isolation and educational campaigns [3, [5].

Mathematical models have become important tools in analyzing the spread and control of infectious diseases. The model formulation process clarifies assumptions, variables parameters. There have been many studies that have mathematically analyzed infectious diseases [4, 7, 15. Recently, many control optimal models pertaining to epidemic disease to epidemic diseases have appeared in the literature. They include, but not limited to, delayed SIRS epidemic model [13, delayed SIR model [1], tuberculosis model [17, HIV model [9] and dengue fever [2].

In this paper, we consider an optimal control problem governed by a system of delay differential equations with general incidence function and time delays. The governing state equations of the optimal control are described in a SIR framework with a general incidence function and a time delays representing the incubation period. Then we derive first-order necessary conditions for existence of the optimal control and develop a numerical method to solve them.

The rest of this paper is organized as follows. In section 2, we give the statement of the optimal control problem. We derive the necessary conditions for existence of the optimal control in section 3. In section 4, we describe the numerical method and present the resulting numerical simulations. Finally, we discuss these results in section 5 along with some concluding remarks. 


\section{Statement of the optimal control problem}

Compute the optimal pair of vaccination and treatment strategies $\left(\mathfrak{u}_{1}, \mathfrak{u}_{2}\right)$ that would maximize the recovered population and minimize both the infected and susceptible population, and at the same time minimize the costs of applying the vaccination and treatment strategies. So we consider the optimal control problem of the form (see Eihab B. M. et al):

$$
\min _{\left(u_{1}, u_{2}\right) \in U} J\left(u_{1}(t), u_{2}(t)\right)=\left\{\begin{array}{l}
S(T)+I(T)-R(T) \\
+\int_{0}^{T}\left(c_{1} u_{1}^{2}(t)+c_{2} u_{2}^{2}(t)+S(t)+I(t)-R(t)\right) d t
\end{array}\right.
$$

subject to the quation

$$
\left\{\begin{array}{l}
\dot{S}=B-\mu_{1} S-f\left(S, I_{\tau}\right)-u_{1} S \\
\dot{I}=f\left(S, I_{\tau}\right)-\left(\mu_{2}+\gamma\right) I-u_{2} I \\
\dot{R}=\gamma I-\mu_{3} R
\end{array}\right.
$$

The two functions $\mathfrak{u}_{1}(t)$ and $\mathfrak{u}_{2}(t)$ represent vaccination and treatment strategies. These control functions are assumed to be $\mathrm{L}^{\infty}(0, \mathrm{~T})$ functions belonging to a set of admissible controls

$$
\mathbb{U}=\left\{\left(u_{1}, u_{2}\right) \in\left(L^{\infty}(0, T)\right)^{2}: u_{1 \min } \leq u_{1}(t) \leq u_{1 \max }, u_{2 \min } \leq u_{2}(t) \leq u_{2 \max }\right\}
$$

where $0 \leq u_{1 \min }<u_{1 \max } \leq 1$ and $0 \leq u_{2 \min }<u_{2 \max } \leq 1$. The two constants $c_{1}$ and $c_{2}$ are weighted cost associated with the use of the controls $u_{1}(t)$ and $u_{2}(t)$, respectively. The state equations are formulated from an SIR model with general incidence model, where $S(t), I(t)$, $R(t)$ are the numbers of susceptible, infected and recovered individuals at time $t$, respectively. The parameters $B$ is the recruitment rate, the death rates for the classes are $\mu_{1}, \mu_{2}$ and $\mu_{3}$, respectively. The average time spent in class I before recovery is $1 / \gamma$. For biological reasons, we assume that $\mu_{1} \leq \mu_{2}+\gamma$; that is, removal of infectives is at least as fast as removal of susceptibles. The time delays $\tau$ represents the incubation period. That is to say, only susceptible individuals who got infected a time $t-\tau$ are able to communicate the disease at time $t$.

As general as possible, the incidence function $f$ must satisfy technical conditions. Thus, we assume that

$\mathbf{H 1} \mathrm{f}$ is non-negative $\mathrm{C}^{1}$ functions on the non-negative quadrant,

H2 for all $(S, I) \in \mathbb{R}_{+}^{2}, f(S, 0)=f(0, I)=0$.

Let us denote by $f_{1}$ and $f_{2}$ the partial derivatives of $f$ with respect to the first and to the second variable

The differential equation model described by (2.2) without controls $\left(u_{1}=u_{2}=0\right)$ has two equilibrium points: a disease-free equilibrium $\mathrm{E}_{0}$ given by

$$
\mathrm{E}_{0}=\left(\frac{\mathrm{B}}{\mu_{1}}, 0,0\right)
$$


and an endemic equilibrium $E^{*}=\left(S^{*}, I^{*}, R^{*}\right)$ where,

$$
\begin{aligned}
\mathrm{S}^{*} & =\frac{\mathrm{B}-\left(\mu_{2}+\gamma\right) \mathrm{I}^{*}}{\mu_{1}} \\
\mathrm{I}^{*} & =\mathrm{I}^{*} \\
\mathrm{R}^{*} & =\frac{\gamma}{\mu_{3}} \mathrm{I}^{*}
\end{aligned}
$$

The basic reproduction number of (2.2) without controls is given by

$$
R_{0}=\frac{f_{2}\left(S^{0}, 0\right)}{\mu_{2}+\gamma}
$$

It was proven that if $R_{0}<1$, then the disease-free equilibrium is asymptotically stable and if $R_{0}>1$ then it is unstable.

On the other-hand, when the controls is not null $\left(u_{1} \neq 0\right.$ and or $\left.u_{2} \neq 0\right)$, we have the SIR model (2.2).

The disease-free equilibrium for system (2.2) is given by

$$
E_{0}^{c}=\left(\frac{B}{\mu_{1}+u_{1}}, 0,0\right)
$$

whereas the endemic equilibrium $E_{c}^{*}$ is given by

$$
\mathrm{E}_{\mathrm{c}}^{*}=\left(\frac{\mathrm{B}-\left(\mu_{2}+\gamma+\mathrm{u}_{2}\right) \mathrm{I}^{*}}{\mu_{1}+\mathrm{u}_{1}}, \mathrm{I}^{*}, \frac{\gamma}{\mu_{3}} \mathrm{I}^{*}\right)
$$

The basic reproduction number $R_{c}$ of system (2.2) is given by

$$
R_{c}=\frac{f_{2}\left(S^{0}, 0\right)}{\mu_{2}+\gamma+u_{2}}
$$

and it is clear that when $u_{1} \rightarrow 0$ and $u_{2} \rightarrow 0$ then $R_{c} \rightarrow R_{0}$

\section{Existence and characterization of the optimal control}

In this section, we discuss the existence of the optimal control and then construct the Hamiltonian of the optimal control problem to derive the first order necessary conditions for the optimal control.

\subsection{Existence of optimal control}

To show the existence of the optimal control for the problem under consideration, we notice that the set of admissible controls $\mathbb{U}$ is, by definition, closed and bounded. It is also convex because $\left[u_{1 \min }, u_{1 \max }\right] \times\left[u_{2 \min }, u_{2 \max }\right]$ is convex in $\mathbb{R}^{2}$. It is obvious that there is an admissible pair $\left(\left(u_{1}(t), u_{2}(t)\right)\right)$ for the problem. Hence, the existence of the optimal control comes as a direct result from the Filippove-Cesari theorem [14. We therefore, have the following result: 
Theorem 3.1. Consider the optimal control problem (2.1) subject to (2.2). Then there exists an optimal pair of controls $\left(\mathrm{u}_{1}^{*}, \mathrm{u}_{2}^{*}\right)$ and a corresponding optimal states $\left(\mathrm{S}^{*}, \mathrm{I}^{*}, \mathrm{R}^{*}\right)$ that minimizes the objective function $\mathrm{J}\left(\mathfrak{u}_{1}, \mathfrak{u}_{2}\right)$ over set of admissible controls $\mathbb{U}$.

Proof. To prove the existence of an optimal control pair, it is important to verify the following assertion.

(1) The set of controls and corresponding state variables is nonempty.

(2) The admissible set $\mathbb{U}$ is convex and closed.

(3) The right-hand side of the state system (2.2) is bounded by a linear function in the state and control variables.

(4) The integrand of the objective functional $L_{S, I, R}\left(u_{1}, u_{2}\right)$ is convex on the set $\mathbb{U}$. The hessian matrix of $L_{S, I, R}\left(u_{1}, u_{2}\right)$ on $\mathbb{U}$ is done by :

$$
M=\left(\begin{array}{cc}
2 c_{1} & 0 \\
0 & 2 c_{2}
\end{array}\right)
$$

$\operatorname{Sp}(M)=\left\{2 c_{1}, 2 c_{2}\right\} \subset \mathbb{R}_{+}^{*}$,

then, $L_{S, I, R}\left(u_{1}, \mathfrak{u}_{2}\right)$ is strictly convex in $\mathbb{U}$.

(5) There exists constants $\omega_{1}>0, \omega_{2}$ and $\rho>1$ such that the integrand $L_{S, I, R}\left(u_{1}, u_{2}\right)$ of the objective functional satisfies

$$
\begin{gathered}
\mathrm{L}_{S, I, R}\left(u_{1}, u_{2}\right) \geq w_{1}\left|\left(u_{1}, u_{2}\right)\right|^{\rho}-w_{2} . \\
L_{S, I, R}\left(u_{1}, u_{2}\right)=c_{1} u_{1}^{2}(t)+c_{2} u_{2}^{2}(t)+S(t)+I(t)-R(t) \\
\geq \min \left(c_{1}, c_{2}\right)\left(u_{1}^{2}(t)+u_{2}^{2}(t)\right)-R(t)
\end{gathered}
$$

$\mathrm{R}(\mathrm{t})$ is bounded because $\mathrm{N}=\mathrm{S}+\mathrm{I}+\mathrm{R}$

i.e

$$
\exists \alpha, \beta, \alpha<\mathrm{R}(\mathrm{t})<\beta, \forall \mathrm{t}
$$

Let $\omega_{1}=\min \left(c_{1}, c_{2}\right)$ and $\omega_{2}=\beta$. We have,

$$
\mathrm{L}_{S, I, R}\left(u_{1}, u_{2}\right) \geq w_{1}\left\|\left(u_{1} ; u_{2}\right)\right\|^{2}-w_{2} .
$$




\subsection{Characterization of optimal control}

In this subsection, we derive the first order necessary conditions for the existence of optimal control, by constructing the Hamiltonian $\mathrm{H}$ and applying the Pontryagin's maximum principle.

To simplify the notations, we write $x(t)=[S(t), I(t), R(t)]^{\top}, u(t)=\left[u_{1}(t), u_{2}(t)\right]^{\top}$ and $\lambda(t)=$ $\left[\lambda_{1}(t), \lambda_{2}(t), \lambda_{3}(t)\right]$. We denote by $g(u(t), x(t))$ the integrand part of the objective function 2.1). With these notations and terminologies, the Hamiltonian is given by

$\mathrm{H}=\mathrm{H}(\mathrm{u}(\mathrm{t}), x(\mathrm{t}), \lambda(\mathrm{t}))$

$H=g(u(t), x(t))+\lambda^{\top}(t) \cdot \dot{x}(t)$

$$
\begin{aligned}
=c_{1} u_{1}^{2} & +c_{2} u_{2}^{2}+S+I-R+\lambda_{1}\left(B-\mu_{1} S-f\left(S, I_{\tau}\right)-u_{1} S\right) \\
& +\lambda_{2}\left(f\left(S, I_{\tau}\right)-\left(\mu_{2}+\gamma\right) I-u_{2} I\right)+\lambda_{3}\left(\gamma I-\mu_{3} R\right) .
\end{aligned}
$$

Let $\chi_{[a, b]}(t)$ be the characteristic function defined by

$$
\chi_{[a, b]}(t)= \begin{cases}1, & \text { if } t \in[a, b], \\ 0, & \text { otherwise. }\end{cases}
$$

Let $\mathrm{u}^{*}=\left[\mathrm{u}_{1}^{*}, \mathrm{u}_{2}^{*}\right]^{\top}$ be the optimal control and $x^{*}(t)=\left[\mathrm{S}^{*}(t), I^{*}(t), R^{*}(t)\right]^{\top}$ be the corresponding optimal trajectory. Then there exists $\lambda(t) \in \mathbb{R}^{3}$ such that the first order necessary conditions for the existence of optimal control are given by the equations

$$
\begin{aligned}
\frac{\partial H}{\partial u}(t) & =0, \\
\frac{d x}{d t}(t) & =\frac{\partial H}{\partial \lambda}, \\
\frac{d \lambda}{d t}(t) & =-\frac{\partial H}{\partial x} .
\end{aligned}
$$

The optimality conditions:

$$
\begin{aligned}
& {\left[\frac{\partial \mathrm{H}}{\partial \mathrm{u}_{1}}(\mathrm{t})\right]_{\mathfrak{u}(\mathrm{t})=\mathfrak{u}^{*}(\mathrm{t})}=0,} \\
& {\left[\frac{\partial \mathrm{H}}{\partial \mathrm{u}_{2}}(\mathrm{t})\right]_{\mathfrak{u}(\mathrm{t})=\mathfrak{u}^{*}(\mathrm{t})}=0 .}
\end{aligned}
$$

Simplifying (3.5) and (3.6), we obtain

$$
\begin{aligned}
& 2 c_{1} u_{1}^{*}-S \lambda_{1}=0, \\
& 2 c_{2} u_{2}^{*}-\lambda_{2} I=0 .
\end{aligned}
$$


Further simplification of (3.8) and (3.9) yields

$$
u_{1}^{*}(t)=\min \left\{u_{1 \max } ; \max \left\{0 ; \frac{S(t) \lambda_{1}(t)}{2 c_{1}}\right\}\right\}
$$

and

$$
u_{2}^{*}(t)=\min \left\{u_{1 \max } ; \max \left\{0 ; \frac{I(t) \lambda_{2}(t)}{2 c_{2}}\right\}\right\} .
$$

The state equations: given by the forms (2.2)

The co-state equations:

$$
\begin{aligned}
\frac{\mathrm{d} \lambda_{1}}{\mathrm{dt}}(\mathrm{t}) & =-\frac{\partial \mathrm{H}}{\partial \mathrm{S}}(\mathrm{t}), \\
\frac{\mathrm{d} \lambda_{2}}{\mathrm{dt}}(\mathrm{t}) & =-\left[\frac{\partial \mathrm{H}}{\partial \mathrm{I}}(\mathrm{t})+\chi_{[0, T-\tau]} \frac{\partial \mathrm{H}}{\partial \mathrm{I}_{\tau}}(\mathrm{t}+\tau)\right], \\
\frac{\mathrm{d} \lambda_{3}}{\mathrm{dt}}(\mathrm{t}) & =-\frac{\partial \mathrm{H}}{\partial \mathrm{R}}(\mathrm{t}),
\end{aligned}
$$

which when simplified, lead to

$$
\begin{aligned}
\frac{d \lambda_{1}}{d t} & =-1+\left(\lambda_{1}(t)-\lambda_{2}(t)\right) f_{1}(S, I)+\left(\mu_{1}+u_{1}\right) \lambda_{1}(t), \\
\frac{d \lambda_{2}}{d t} & =-1+\left(\lambda_{1}(t+\tau)-\lambda_{2}(t+\tau)\right) x_{[0, T-\tau]}(t) f_{2}(S, I)+\left(\mu_{2}+\gamma+u_{2}\right) \lambda_{2}(t)-\gamma \lambda_{3}(t), \\
\frac{d \lambda_{3}}{d t} & =1+\mu_{3} \lambda_{3}(t) .
\end{aligned}
$$

The transversality conditions:

$$
\begin{aligned}
& \lambda_{1}(\mathrm{~T})=1, \\
& \lambda_{2}(\mathrm{~T})=1, \\
& \lambda_{3}(\mathrm{~T})=-1 .
\end{aligned}
$$

Remark 3.2. It is noting that

1. The Hamiltonian function $\mathrm{H}$ is strongly convex in the control variables.

2. The right-hand sides of the state and co-state equations are Lipschitz continuous.

3. The set of the admissible controls $\mathbb{U}$ is convex

\section{Numerical simulations}

In this section, we apply the above optimal control theory with consideration of its applicability. we discuss the discretization of the optimal control problem described and present the numerical 
results obtained through our simulations. The algorithm describing the approximation method to obtain the optimal control is the following algorithm inspired from [13. The Algorithm used here is a numerical variation of forward Euler method with a step size $h$. We explicitly write the forward Euler method for the state and the adjoint.

step1: for $i=-m, \ldots, 0$, do :

$S_{i}=S_{0} ; I_{i}=I_{0} ; R_{i}=R_{0} ; u_{1}^{i}=0 ; u_{2}^{i}=0$

end for

for $i=n, \ldots, n+m$

$\lambda_{1}^{i}=1 ; \lambda_{2}^{i}=1 ; \lambda_{3}^{i}=-1$

end for

step2 :for $i=0, \ldots, n-1$

$S_{i+1}=S_{i}+h\left(B-\mu_{1} S_{i}-\beta S_{i} I_{i}-u_{1}^{i} S_{i}\right)$

$I_{i+1}=I_{i}+h\left(\beta S_{i} I_{i}-\left(\mu_{2}+\gamma\right) I_{i}-u_{2}^{i} I_{i}\right)$

$R_{i+1}=R_{i}+h\left(\gamma I_{i}-\mu_{3} R_{i}\right)$

$\lambda_{1}^{n-i-1}=\lambda_{1}^{n-i}-h\left(-1+\left(\lambda_{1}^{n-i}-\lambda_{2}^{n-i}\right) \beta I_{i+1}+\left(\mu_{1}+u_{1}^{i}\right) \lambda_{1}^{n-i}\right.$

$\lambda_{2}^{n-i-1}=\lambda_{2}^{n-i}-h\left(-1+\left(\lambda_{1}^{n+m-i}-\lambda_{2}^{n+m-i}\right) \chi_{[0, T-\tau]}\left(t_{n-i}\right) \beta S_{i+1}\right.$

$$
\left.+\left(\mu_{2}+\gamma+u_{2}^{i}\right) \lambda_{2}^{n-i}-\gamma \lambda_{3}^{n-i}\right)
$$

$\lambda_{3}^{n-i-1}=\lambda_{3}^{n-i}-h\left(1+\mu_{3} \lambda_{3}^{n-i}\right)$

$u_{1}^{i+1}=S_{i+1} \lambda_{1}^{n-i} / 2 c_{1}$

$u_{2}^{i+1}=I_{i+1} \lambda_{2}^{n-i} / 2 c_{2}$

end for

step3 :for $i=1, \ldots, n$, write

$S^{*}\left(t_{i}\right)=S_{i}, \quad I^{*}\left(t_{i}\right)=I_{i} \quad R^{*}\left(t_{i}\right)=R_{i} \quad u_{1}^{*}\left(t_{i}\right)=u_{1}^{i} \quad$ and $\quad u_{2}^{*}\left(t_{i}\right)=u_{2}^{i}$. 


\section{Comments}

Fig 1. represent the different dynamics of the susceptible population for different aspect of control. The Orange color represent the population when there is treatment but not vaccination $\left(u_{1}=0\right.$ and $u_{2} \neq 0$ ). The blue curve represent the population when there are vaccination and treatment $\left(u_{1} \neq 0\right.$ and $\left.u_{2} \neq 0\right)$. The green curve show the evolution of the susceptible population when there is just treatment but not vaccination $\left(u_{1}=0\right.$ and $\left.u_{2} \neq 0\right)$. This show that, without vaccination so many people are exposed to disease.

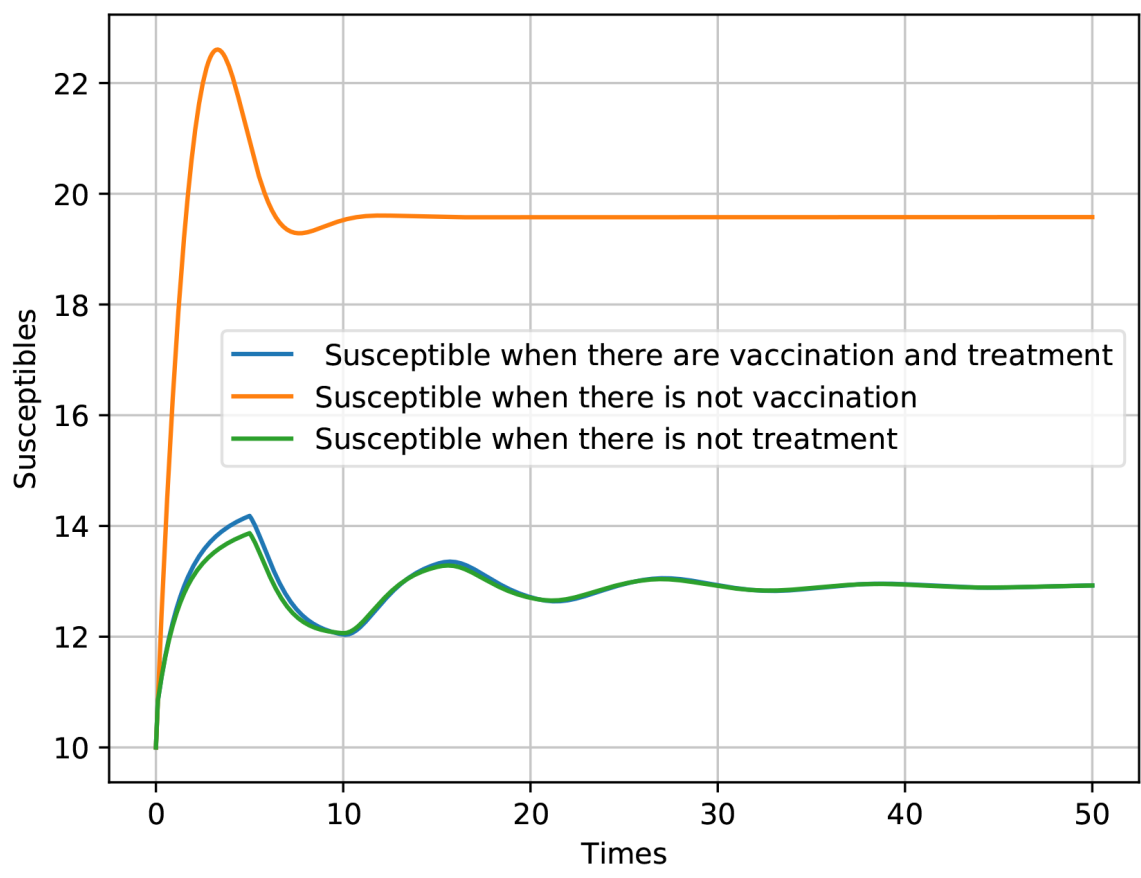

Fig 1. Dynamic of susceptible population with different aspect of control. 
Fig 2. represent the different dynamics of the infected population for different aspect of control. The Orange color present the evolution of the infected population when there is treatment but not vaccination $\left(u_{1}=0\right.$ and $\left.u_{2} \neq 0\right)$. The blue curve represent the population when there are vaccination and treatment $\left(u_{1} \neq 0\right.$ and $\left.u_{2} \neq 0\right)$. The green curve show the evolution of the infected population when there is just treatment but not vaccination $\left(u_{1}=0\right.$ and $\left.u_{2} \neq 0\right)$. Naturally, as many people are exposed to the disease without vaccination, we see the growth of the infected population.

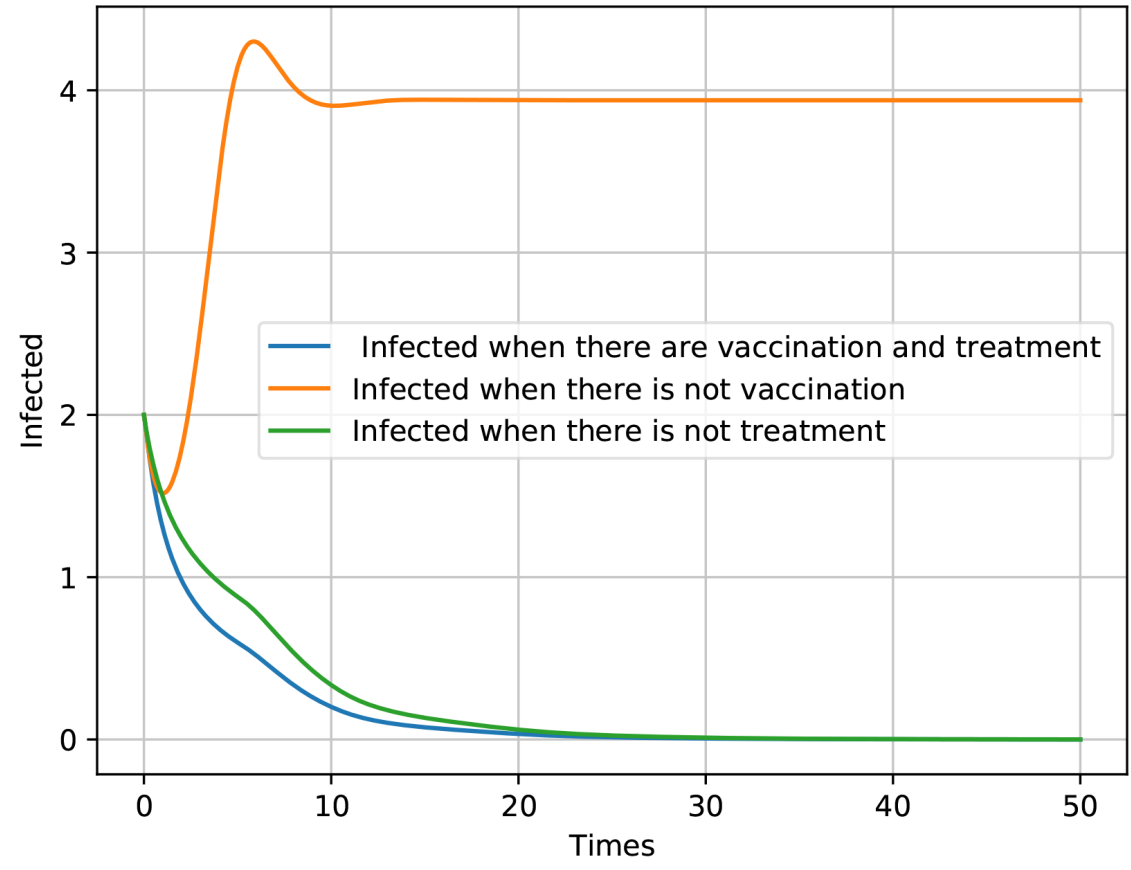

Fig 2. Dynamic of infected population with different aspect of control. 
Fig 3. represent the different dynamics of the infected population for different aspect of control. The Orange color present the evolution of the recovered population when there is treatment but not vaccination $\left(u_{1}=0\right.$ and $\left.u_{2} \neq 0\right)$. The blue curve represent the population when there are vaccination and treatment $\left(u_{1} \neq 0\right.$ and $\left.u_{2} \neq 0\right)$. The green curve show the evolution of the recovered population when there is just treatment but not vaccination $\left(u_{1}=0\right.$ and $\left.u_{2} \neq 0\right)$. As many people are exposed to the disease without vaccination, indeed we see the growth of the recovered population.

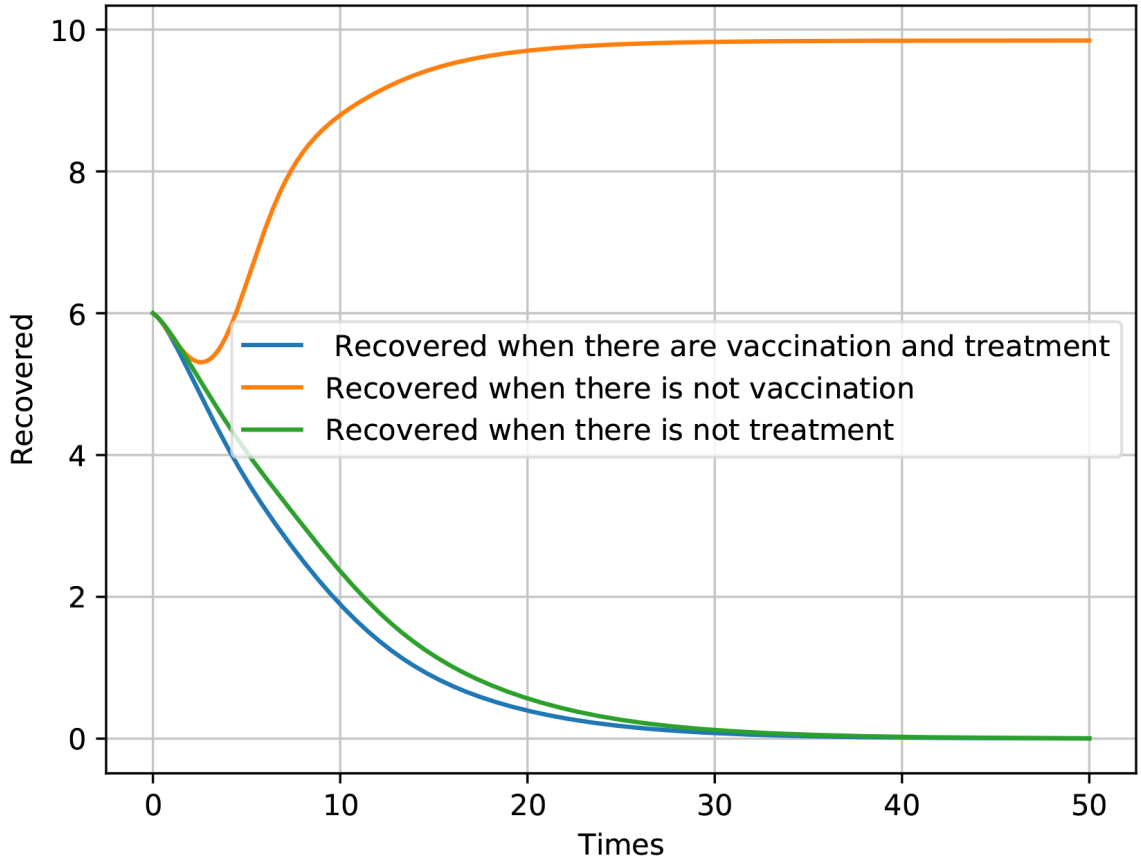

Fig 3. Dynamic of recovered population with different aspect of control.

In conclusion, observing the figures, we can deduce that the strategy leading to the vaccination alone $\left(u_{1} \neq 0\right.$ and $\left.u_{2}=0\right)$ should be preferable to the joint use of vaccination $\left(u_{1} \neq 0\right)$ and treatment $\left(u_{2} \neq 0\right)$. The optimal control strategy here shows that prevention is more effective for the eradication of the disease. 


\section{Conclusion}

In this paper, we considered an optimal control problem for a SIR model with time delay (representing the incubation period ) and general incidence function. The main idea developed here is the optimal control in epidemics in order to search among the available strategies, the most effiscience one that reduce the infection rate to a minimum level while optimizing the cost deploying a therapy and preventive vaccine that is used to control the disease progression. The two control functions $u_{1}(t)$ and $u_{2}(t)$, which represent the vaccination and the treatment strategies are subject to time delays before being effective. Then we formulated the objective function of the optimal control problem. We discussed the existence of the optimal control and then derived the first order necessary conditions for the optimal control through constructing the Hamiltonian and using the Pontryagin's maximum principle to achieve our aim. Finally, to end our study, we do a numerical simulation to corroborate the theoretical results obtained.

\section{Acknowledgments}

The authors want to thank the anonymous referee for his valuable comments on the paper.

\section{Competing interests}

The authors declare that they have no competing interests.

\section{Author's contribution}

Aboudramane Guiro provide the subject, wrote the introduction and the conclusion and verified some calculation. Moussa BARRO conceived the study and computed the equilibria and their local stabilities. Dramane OUEDRAOGO wrote mathematical formula, he bring up the control strategy and did all the calculus with the second author. All the authors read and approved the final manuscript.

\section{References}

[1] A. Abta, H. Laarabi, H. T. Alaoui , The hopf bifurcation analysis and optimal Control of a delayed SIR epidemic model, Int. J. Anal. (2014), 1-10.

[2] D. Aldila, T. Gotz, E. Soewono, An optimal Control problem arising from a dengue disease transmission model, Math. Biosci. 242 (1)(2013), 9-16.

[3] F. G. Ball, E. S. Knock, P.D. O'Neil Control of emerging infectious diseases using responsive imperfect vaccination and isolation, Math. Biosci. 216 (1) (2008), 100-113.

[4] N. Becker, The use of epidemic models, Biometrics 35(1978) 295-305. 
[5] C. Castilho, Optimal Control of an epidemic through educational campaigns, Electron. J. Differ. Equ. 2006 (2006), 1-11.

[6] C. Chiyaka, W. Garira, S. Dube, Transmission model of endemic human malaria in a partially immune population. Math. Comput. Model. 46 (2007), 806-822.

[7] K. Dietz, The first epidemic model: a historical note on P.D. En'ko. Aust. J. Stat. 30A (1988), $56-65$.

[8] H. Gaff, E.Schaefer, Optimal control applied to vaccination and treatment strategies for various epidemiological models, Math. Biosci. Eng. 6 (3) (2009), 469-492.

[9] K. Hattaf, N. Yousfi, Optimal Control of a delayed HIV infection model with immune response using an efficient numerical method, Int. Sch. Res. Netw. (2012) (2012), 1-7.

[10] H. W. Hethcote, P. van den Driessche; Some epidemiological models with nonlinear incidence. J.Math. Biol. 29 (1991), 271-287.

[11] H. W. Hethcote, The mathematics of infectious, SIAM Rev.42 (2000), 599-653.

[12] A. Kaddar; On the dynamics of a delayed SIR epidemic model with a modified saturated incidence rate, Electron. J. Differ. Equ. 13 (2009), 1-7.

[13] H. Laarabi, A. Abta, K. Hattaf , Optimal Control of a delayed SIRS epidemic model with vaccination and treatment, Acta Biotheor. 63 (15) (2015), 87-97.

[14] S. Nababan ; A filippov-type lemma for functions involving delays and its application to timedelayed optimal control problems,optim. Theory appl. 273 (1979), 357-376.

[15] P. Ogren, C. F. Martin, Vaccination strategies for epidemics in highly mobile populations. Appl. Math. Comput. 127 (2002), 261-276.

[16] S. Ruan, D. Xiao, J. C. Beier; On the delayed ross-macdonald model for malaria transmission, Bull. Math. Biol. 70 (2008), 1007-1025.

[17] C. J. Silva, D. F. Torres, Optimal Control strategies for tuberculosis treatment: a case study in angola, Numer. Algebra Control Optim. 2 (3) (2012), 601-617.

[18] G. Zaman, Y.H. Kang, J.H. Jung, Optimal treatment of an SIR epidemic model with time delay, Biosystems 98 (1) (2009), 43-50. 